\title{
Impacto da Insuficiência Renal Aguda na Evolução Hospitalar Após Tratamento Percutâneo do Infarto Agudo do Miocárdio
}

\author{
Luciano Nunes dos Santos ${ }^{1}$, Fabio Conejo ${ }^{2}$, Francisco Hedilberto Feitosa Filho ${ }^{3}$, \\ Carlos Augusto Homem de Magalhães Campos ${ }^{4}$, J. Mariani Junior ${ }^{5}$, Celso Kiyochi Takimura ${ }^{6}$, \\ Antônio Esteves Filho ${ }^{7}$, Expedito Eustáquio Ribeiro da Silva ${ }^{8}$, \\ Roberto Kalil Filho ${ }^{9}$, Pedro Alves Lemos Neto ${ }^{10}$
}

\section{RESUMO}

Introdução: A insuficiência renal aguda (IRA) é uma complicação possível após intervenção coronária percutânea (ICP). O objetivo deste estudo foi avaliar a ocorrência e o impacto prognóstico da IRA pós-ICP em pacientes com infarto agudo do miocárdio com supradesnivelamento do segmento ST (IMCSST). Métodos: Registro unicêntrico, que analisou a evolução hospitalar de 501 pacientes admitidos com IMCSST submetidos à ICP primária, de resgate ou tardia. Foram avaliados a incidência e os preditores de IRA pós-ICP. Resultados: A idade média foi $60,7 \pm 12,6$ anos e $67 \%$ eram do gênero masculino. A população apresentava características de alto risco cardiovascular, sendo $30 \%$ diabéticos e 7,4\% com doença renal crônica (DRC) preexistente. A artéria descendente anterior foi a principal artéria culpada (49,4\%) e 15\% dos pacientes se apresentaram em Killip III ou IV. A IRA ocorreu em $24,7 \%$ dos pacientes, que, quando comparados àqueles sem IRA, eram significativamente mais idosos, diabéticos, com DRC e insuficiência cardíaca, além de apresentarem maior elevação enzimática e menor fração de ejeção. A mortalidade hospitalar foi maior nos pacientes que desenvolveram IRA $(29 \%$ vs. $4,8 \%$; $P<0,01)$. Os preditores independentes

\section{ABSTRACT}

\section{Impact of Acute Renal Failure on In-Hospital Outcomes Following Percutaneous Treatment of Acute Myocardial Infarction}

Background: Acute renal failure (ARF) is a possible complication after percutaneous coronary intervention $(\mathrm{PCl})$. The objective of this study was to evaluate the occurrence and prognostic impact of $\mathrm{ARF}$ after $\mathrm{PCl}$ in patients with $\mathrm{ST}$ segment elevation myocardial infarction (STEMI). Methods: Single-center registry evaluating in-hospital outcomes of 501 patients admitted with STEMI undergoing primary, rescue or late $\mathrm{PCI}$. The incidence and predictors of ARF after PCI were evaluated. Results: Mean age was $60.7 \pm 12.6$ years and $67 \%$ were male. The population had high cardiovascular risk characteristics, with $30 \%$ of diabetics and $7.4 \%$ with preexisting chronic kidney disease (CKD). The left anterior descending artery was the culprit vessel in $49.4 \%$ of the cases and $15 \%$ of patients had Killip class III or IV. ARF was observed in $24.7 \%$ of patients, who were significantly older, had more diabetes, history of CKD or heart failure, had higher enzyme elevation and lower ejection fraction when compared to those without ARF. In-hospital
1 Cardiologista intervencionista do Instituto do Coração do Hospital das Clínicas da Faculdade de Medicina da Universidade de São Paulo. São Paulo, SP, Brasil.

${ }^{2}$ Cardiologista intervencionista do Instituto do Coração do Hospital das Clínicas da Faculdade de Medicina da Universidade de São Paulo. São Paulo, SP, Brasil.

${ }^{3}$ Cardiologista intervencionista do Instituto do Coração do Hospital das Clínicas da Faculdade de Medicina da Universidade de São Paulo. São Paulo, SP, Brasil.

${ }^{4}$ Cardiologista intervencionista do Instituto do Coração do Hospital das Clínicas da Faculdade de Medicina da Universidade de São Paulo. São Paulo, SP, Brasil.

${ }^{5}$ Cardiologista intervencionista do Instituto do Coração do Hospital das Clínicas da Faculdade de Medicina da Universidade de São Paulo. São Paulo, SP, Brasil.

${ }^{6}$ Doutor. Médico assistente no Hospital da Luz, São Paulo, SP, Brasil. 7 Cardiologista intervencionista do Instituto do Coração do Hospital das Clínicas da Faculdade de Medicina da Universidade de São Paulo. São Paulo, SP, Brasil.

\begin{abstract}
${ }^{8}$ Livre-docente. Cardiologista intervencionista do Instituto do Coração do Hospital das Clínicas da Faculdade de Medicina da Universidade de São Paulo. São Paulo, SP, Brasil.

${ }^{9}$ Professor titular. Diretor da Divisão de Cardiologia do Instituto do Coração do Hospital das Clínicas da Faculdade de Medicina da Universidade de São Paulo. São Paulo, SP, Brasil.

${ }^{10}$ Livre-docente. Diretor do Serviço de Hemodinâmica e Cardiologia Intervencionista do Instituto do Coração do Hospital das Clínicas da Faculdade de Medicina da Universidade de São Paulo. São Paulo, SP, Brasil.
\end{abstract}

Correspondência: Pedro Alves Lemos Neto. Av. Dr. Enéas Carvalho de Aguiar, 44 - Jardim Paulista - São Paulo, SP, Brasil - CEP 05403-000 E-mail: pedro.lemos@incor.usp.br

Recebido em: 20/9/2013 • Aceito: 3/12/2013 
de IRA foram idade > 76 anos, DRC prévia, Killip III ou IV, necessidade de cirurgia vascular ou transfusão sanguínea. Conclusões: A disfunção renal aguda após ICP no IMCSST foi uma complicação frequente e associada com aumento da mortalidade hospitalar.

DESCRITORES: Insuficiência renal. Intervenção coronária percutânea. Infarto do miocárdio.

A doença arterial coronária é uma das principais causas de mortalidade em países desenvolvidos. Apesar da mortalidade após o infarto agudo do miocárdio (IAM) ter sido reduzida nas últimas décadas, ainda existem subgrupos de pacientes que apresentam maior risco de complicações e óbito, como aqueles com disfunção renal. ${ }^{1-3}$

Pacientes submetidos à intervenção coronária percutânea (ICP) pós-IAM representam uma população de elevado risco para o desenvolvimento de insuficiência renal aguda (IRA), uma vez que diversas condições podem favorecer sua ocorrência, como a hipotensão arterial, a disfunção ventricular, o uso de fármacos com potencial nefrotóxico e a impossibilidade de iniciar medidas profiláticas no contexto da emergência. Alguns estudos mostram que a disfunção renal é um preditor de risco independente de morte no IAM. ${ }^{4-6} \mathrm{~A}$ ICP apresenta ainda o potencial de ocasionar disfunção renal induzida pela utilização do meio de contraste. Sua ocorrência em exames diagnósticos e terapêuticos tem sido relacionada com aumento na morbidade e na mortalidade hospitalar, aumento do tempo de hospitalização, desenvolvimento de disfunção renal crônica e diálise. ${ }^{7-9}$

O objetivo deste estudo foi avaliar o impacto da IRA na evolução hospitalar de pacientes com IAM com supradesnivelamento do segmento ST (IMCSST) tratados por via percutânea.

\section{MÉTODOS}

\section{População do estudo}

Foram avaliados pacientes com IMCSST submetidos à ICP primária, ICP de resgate ou ICP tardia, em um único centro, o Instituto do Coração do Hospital das Clínicas da Faculdade de Medicina da Universidade de São Paulo (InCor/HCFMUSP), em São Paulo (SP), no período de janeiro de 2008 a março de 2011.

\section{Coleta dos dados}

Os dados da evolução hospitalar foram coletados prospectivamente durante a internação índice, seguindo o preenchimento de formulários previamente padronizados, com a assinatura de termo de consentimento mortality was higher in patients who developed ARF $(29 \%$ vs. $4.8 \% ; \mathrm{P}<0.01)$. Independent predictors of ARF were age $>76$ years, previous CKD, Killip class III or IV, need of vascular surgery or blood transfusion. Conclusions: Acute renal failure after $\mathrm{PCl}$ in STEMI was a frequent complication and was associated with increased in-hospital mortality.

DESCRIPTORS: Renal insufficiency. Percutaneous coronary intervention. Myocardial infarction.

livre e esclarecido de participação em estudo de ICP. A coleta incluiu características clínicas, resultados de exames laboratoriais, e dados do procedimento invasivo e da evolução clínica até a alta hospitalar. Foram analisados pacientes que desenvolveram IRA, com ou sem antecedente de doença renal crônica.

\section{Procedimento}

Em todos os procedimentos, foi administrada previamente heparina não fracionada, na dose de $70 \mathrm{U} / \mathrm{kg}$ a $100 \mathrm{U} / \mathrm{kg}$, para que fosse obtido o tempo de coagulação ativada entre 250 e 300 segundos, exceto naqueles que já estavam em uso de heparina (de baixo peso molecular ou não fracionada) na admissão da Hemodinâmica, quando a dose era ajustada de acordo com a meia-vida do fármaco utilizado ou com os resultados do tempo de coagulação ativado. Os pacientes utilizaram dupla terapia de antiagregação plaquetária com ácido acetilsalicílico e clopidogrel. Durante o procedimento diagnóstico e terapêutico, houve a preocupação em minimizar o volume de contraste e evitar fármacos com potencial nefrotóxico. A critério do médico assistente e conforme a condição clínica, os paciente recebiam hidratação com soro fisiológico periprocedimento.

A escolha da via de acesso, as técnicas, os materiais e os métodos diagnósticos e de intervenção foram de livre escolha dos operadores. Todas as angiografias foram analisadas pelo Serviço de Hemodinâmica e Cardiologia Intervencionista do InCor/HCFMUSP. A aquisição de imagens foi realizada usando duas ou mais projeções angiográficas da estenose após a administração de vasodilatadores.

\section{Definições}

O diagnóstico de IMCSST foi realizado na ocorrência de elevação persistente de ST > $1 \mathrm{~mm}$ em duas derivações contíguas, ou bloqueio de ramo esquerdo novo ao eletrocardiograma em paciente com quadro clínico sugestivo de isquemia miocárdica.

IRA foi definida como elevação de $25 \%$ da creatinina sérica basal ou aumento absoluto de $0,5 \mathrm{mg} / \mathrm{dL}$ de creatinina sérica avaliada nos primeiros 7 dias após a realização do procedimento. ${ }^{10}$ Os exames iniciais de creatinina foram obtidos a partir de valores prévios no 
prontuário do paciente ou de exames coletados na sala de emergência no momento da admissão hospitalar.

A ICP foi definida como primária quando o procedimento foi realizado na fase aguda do IAM $(<12$ horas ou entre 12 e 24 horas na presença de sintomas) utilizando cateter balão ou stent, sem uso prévio de fibrinolítico, com o objetivo de restabelecer o fluxo coronário anterógrado. Considerou-se ICP de resgate aquela realizada quando o agente fibrinolítico não obteve sucesso na reperfusão do músculo isquêmico (resolução do supradesnivelamento do ST < 50\% nos primeiros 60 minutos da administração). A ICP foi definida como tardia quando realizada $>24$ horas após o início dos sintomas.

O óbito foi definido como morte por qualquer causa. As complicações vasculares avaliadas foram pseudoaneurisma, fístula arteriovenosa, hematoma significativo no local do acesso (definido pelo diâmetro $\geq 10 \mathrm{~cm}$ ) e sangramento significativo (definido como queda de hemoglobina $>2 \mathrm{~g} / \mathrm{dL}$ ou que necessitou de transfusão sanguínea).

\section{Análise estatística}

As variáveis contínuas foram descritas como média \pm desvio padrão e comparadas com o teste de Wilcoxon. As variáveis categóricas foram representadas como porcentual, sendo comparadas com o teste qui-quadrado. As características clínicas e angiográficas apresentadas foram incluídas em um modelo de regressão para detecção dos preditores de IRA após o procedimento.

Todos os testes foram bicaudais e um valor de $\mathrm{P}<0,05$ foi considerado significativo. As análises foram realizadas utilizando o programa Statistical Package for the Social Sciences (SPSS), versão 17.0 (SPSS Inc., Chicago, Estados Unidos).

\section{RESULTADOS}

No período de janeiro de 2008 a março de 2011, foram avaliados 501 pacientes consecutivamente admitidos com IMCSST, sendo suas características clínicas sumarizadas na Tabela 1. Os pacientes foram predominantemente do sexo masculino $(67 \%)$, com média de idade de 60,7 \pm 12,6 anos e 29,7\% eram diabéticos. Doença renal crônica foi observada em 7,4\% da população do estudo. Em relação ao tipo de procedimento, a ICP primária foi realizada em 59,2\%, ICP de resgate em $15,3 \%$ e ICP tardia em $25,5 \%$ dos casos. O tempo entre o início dos sintomas e a admissão hospitalar (tempo dor-porta) foi de 5 horas e 12 minutos, sem diferença estatística entre os pacientes que desenvolveram IRA e aqueles que mantiveram a função renal normal $(P=0,27)$. O território da artéria descendente anterior foi a localização mais relacionada ao infarto $(49,4 \%)$, seguido pela artéria coronária direita (39,8\%) e a circunflexa $(10,8 \%)$. Em relação à apresentação hemodinâmica na admissão hospitalar, a maioria dos pacientes encontrava-se em Killip I (75\%); contudo, cerca de $15 \%$ dos pacientes já se apresentaram em Killip III ou IV. Os inibidores da glicoproteína IIb/IIla foram utilizados em aproximadamente um terço dos casos.

A IRA após a ICP ocorreu em 124 (24,7\%) pacientes. Comparativamente, os pacientes que evoluíram com IRA eram mais idosos, diabéticos e tinham mais frequentemente antecedente de doença renal crônica e insuficiência cardíaca. Nesse grupo, observaram-se ainda uma menor fração de ejeção do ventrículo esquerdo $(40,2 \%$ vs. 47\%; P < 0,01) e uma maior elevação de enzimas cardíacas (pico CKMB: 255,8 UI/L vs. $213,3 \mathrm{UI} / \mathrm{L} ; P=0,01)$. Não houve diferença entre os dois grupos com relação ao tempo de início dos sintomas e a admissão hospitalar (tempo dor-porta) ou ao tipo de ICP realizada (primária, de resgate ou tardia), conforme observado na Tabela 1.

Com relação aos desfechos hospitalares, observou-se que os pacientes com IRA apresentaram mortalidade significativamente maior que os pacientes que não apresentaram perda da função renal (29\% vs. $4,8 \% ; \mathrm{P}<0,01)$. Dentre os óbitos, $87,3 \%$ foram de causa cardiovascular e $34 \%$ dos pacientes tiveram complicações infecciosas que contribuíram para o desfecho. Observamos, ainda, que os pacientes com IRA tiveram uma maior necessidade de transfusões, sem aumento significativo na incidência de fístulas, pseudoaneurisma ou sangramentos no local do acesso (Tabela 2). Houve também tendência, embora não estatisticamente significativa, de maior incidência de acidente vascular cerebral hemorrágico $(1,6 \%$ vs. $0 \%$; $\mathrm{P}=0,06)$. Entre os pacientes que evoluíram com IRA, cerca de $12 \%$ necessitaram realizar diálise durante a internação hospitalar.

Foram identificados como preditores independentes de ocorrência de IRA durante a internação: idade $>76$ anos, antecedente de doença renal crônica, apresentação inicial em Killip III ou IV, realização de cirurgia vascular ou necessidade de transfusão sanguínea (Tabela 3). Os pacientes que apresentavam a função sistólica do ventrículo esquerdo preservada, com fração de ejeção $>60 \%$, tiveram menor ocorrência de IRA.

\section{DISCUSSÃO}

A IRA é uma possível complicação após procedimentos diagnósticos ou terapêuticos coronários e seu desenvolvimento tem sido associado com hospitalização prolongada, disfunção renal persistente, e mortalidade precoce e tardia. Estudos clínicos observacionais têm demonstrado que a disfunção renal é um fator de risco independente para morte em pacientes com IAM. . $^{4-6,11} \mathrm{No}$ registro GRACE, o aumento de $1 \mathrm{mg} / \mathrm{dL}$ na creatinina inicial elevou o risco para óbito hospitalar em $20 \%{ }^{12}$

Nosso estudo observou que a IRA é uma complicação frequente após ICP de pacientes internados com 
TABELA 1

Características clínicas e do procedimento

\begin{tabular}{|c|c|c|c|c|}
\hline Características & $\begin{array}{c}\text { Global } \\
(n=501)\end{array}$ & $\begin{array}{c}\text { IRA } \\
(n=124)\end{array}$ & $\begin{array}{l}\text { Sem IRA } \\
(\mathrm{n}=377)\end{array}$ & Valor de $\mathbf{P}$ \\
\hline Gênero masculino, \% & 67,8 & 67,9 & 67,8 & 0,90 \\
\hline Idade, anos & $60,7 \pm 12,6$ & $65,6 \pm 12,9$ & $59,1 \pm 12,1$ & $<0,01$ \\
\hline Diabetes, $\%$ & 29,7 & 38,7 & 26,8 & 0,03 \\
\hline Hipertensão arterial, \% & 75,5 & 81,5 & 73,5 & 0,17 \\
\hline Dislipidemia, \% & 70,3 & 72,6 & 69,8 & 0,25 \\
\hline Tabagismo atual, $\%$ & 42,6 & 35,5 & 44,8 & 0,09 \\
\hline AF DAC precoce, $\%$ & 29,5 & 21,8 & 32,1 & 0,07 \\
\hline IRC prévia, \% & 7,4 & 20,2 & 3,2 & $<0,01$ \\
\hline Transplante renal prévio, \% & 0,8 & 0,8 & 0,8 & $>0,99$ \\
\hline DAOP, $\%$ & 7 & 12,1 & 5,3 & 0,03 \\
\hline Insuficiência cardíaca, \% & 12,4 & 21 & 9,5 & $<0,01$ \\
\hline ICP prévia, \% & 13,9 & 12,1 & 14,6 & 0,72 \\
\hline RM prévia, \% & 5,8 & 9,7 & 4,5 & 0,09 \\
\hline Killip & & & & $<0,01$ \\
\hline I & 75,2 & 45,5 & 85,1 & \\
\hline$\|$ & 9 & 12,2 & 8 & \\
\hline III & 3,8 & 10,6 & 1,6 & \\
\hline IV & 12 & 31,7 & 5,3 & \\
\hline Tempo dor-porta, minutos & $312 \pm 272$ & $349 \pm 288$ & $301 \pm 249$ & 0,27 \\
\hline Artéria tratada, \% & & & & 0,07 \\
\hline DA & 49,4 & 54,0 & 47,8 & \\
\hline$C D$ & 39,8 & 39,5 & 40,0 & \\
\hline$C x$ & 10,8 & 6,5 & 12,2 & \\
\hline FEVE, \% & $45,7 \pm 11,6$ & $40,2 \pm 10,7$ & $47,6 \pm 11,3$ & $<0,01$ \\
\hline Tipo de ICP, \% & & & & 0,40 \\
\hline Primária & 59,2 & 61,3 & 58,3 & \\
\hline Resgate & 15,3 & 15,3 & 15,4 & \\
\hline Tardia & 25,5 & 23,4 & 26,3 & \\
\hline Uso de GP Ilb/lla, \% & 33,1 & 30,6 & 33,9 & 0,50 \\
\hline
\end{tabular}

TABELA 2

Desfechos e complicações intra-hospitalares

\begin{tabular}{|c|c|c|c|c|}
\hline Eventos & $\begin{array}{c}\text { Global } \\
(n=501)\end{array}$ & $\begin{array}{c}\text { IRA } \\
(n=124)\end{array}$ & $\begin{array}{r}\text { Sem IRA } \\
(n=377)\end{array}$ & Valor de $\mathrm{P}$ \\
\hline Mortalidade, $\%$ & 10,8 & 29 & 4,8 & $<0,01$ \\
\hline AVC hemorrágico, $\%$ & 0,4 & 1,6 & 0 & 0,06 \\
\hline Pseudoaneurisma,\% & 1,6 & 1,6 & 1,6 & $>0,99$ \\
\hline Cirurgia vascular, $\%$ & 1,7 & 4,0 & 1,1 & 0,03 \\
\hline Sangramento via de acesso $\%$ & 3,7 & 5,6 & 3,1 & 0,16 \\
\hline Transfusão,\% & 2,8 & 8,1 & 1,1 & $<0,01$ \\
\hline
\end{tabular}


TABELA 3

Preditores independentes de insuficiência renal aguda $(n=501)$

\begin{tabular}{lccc}
\hline Preditores & Odds Ratio & IC 95\% & Valor de P \\
\hline Idade $>$ 76 anos & 2,54 & $1,44-4,50$ & $<0,01$ \\
DRC & 6,47 & $2,45-17,09$ & $<0,01$ \\
FEVE $>$ 60\% & 0,95 & $0,93-0,98$ & $<0,01$ \\
Killip III & 7,07 & $1,87-26,69$ & $<0,01$ \\
Killip IV & 6,53 & $3,20-13,33$ & $<0,01$ \\
Transfusão sanguínea & 4,51 & $1,01-20,16$ & 0,05 \\
Cirurgia vascular & 7,45 & $1,61-34,54$ & 0,01 \\
\hline
\end{tabular}

$\mathrm{DRC}=$ doença renal crônica; FEVE = fração de ejeção do ventrículo esquerdo.

IMCSST, ocorrendo em quase um quarto dos pacientes avaliados. Sadeghi et al. ${ }^{6}$ e Lanza e Passos et al. ${ }^{13}$ encontraram IRA em pacientes pós-IAM em 18\% e 15,3\%, respectivamente. Observamos, em nosso trabalho, que os principais preditores independentes da ocorrência de IRA foram a idade > 76 anos, antecedente de doença renal crônica, apresentação hemodinâmica em Killip III ou IV, necessidade de realização de cirurgia vascular ou de transfusões sanguíneas. A presença de fração de ejeção > 60\% foi um fator independente de proteção para IRA. Marenzi et al., ${ }^{14}$ estudando 208 pacientes com IAM, observaram incidência de IRA de $19 \%$ pós-ICP, relatando um risco elevado entre os pacientes mais velhos, com disfunção renal à admissão hospitalar e maior aumento enzimático - achados estes semeIhantes ao observado em nosso estudo. Observaram, ainda, um maior risco para os pacientes com menor fração de ejeção do ventrículo esquerdo, maior atraso para reperfusão miocárdica e naqueles com IAM de parede anterior, possivelmente relacionado ao maior volume de contraste relatado pelo pesquisador para tratar esse território e/ou pelo maior comprometimento hemodinâmico.

A piora da função renal tem sido associada com um aumento no risco de sangramentos e de complicações relacionadas ao acesso vascular após a ICP. ${ }^{15}$ Alguns estudos sugerem uma relação entre a ocorrência de sangramentos e um aumento na mortalidade em pacientes com IAM. ${ }^{16,17}$ No contexto da ICP primária, no qual existe um risco particularmente elevado devido à necessidade de anticoagulação e antiagregação plaquetária agressiva, a incidência de complicações vasculares, nesse subgrupo de pacientes com disfunção renal, permanece incerta. ${ }^{18}$ No presente estudo, não observamos aumento significativo de complicações relacionado ao acesso arterial (pseudoaneurismas, fístulas ou sangramento significativo no local do acesso) em pacientes que apresentaram piora da função renal após a intervenção coronária: 8,0\% nos pacientes com IRA e 4,7\% naqueles sem disfunção renal. Contudo, a necessidade de transfusão sanguínea por outros sangramentos mostrou-se preditora independente de insuficiência renal nestes pacientes $(\mathrm{OR}=4,51$; IC 95\% 1,01-20,16; $P=0,05)$.

Em nosso estudo, a mortalidade hospitalar foi significativamente superior nos pacientes que desenvolveram perda da função renal após a ICP (29\% vs. 4,8\%, $P<0,01)$. Da mesma forma, diversos autores encontraram significativo aumento de mortalidade precoce e tardia nos pacientes com perda da função renal pós-ICP no IAM. ${ }^{6,11,14,19}$ Rihal et al., ${ }^{9}$ definindo IRA como um aumento na creatinina $\geq 0,5 \mathrm{mg} / \mathrm{dL}$, observaram que os pacientes com disfunção renal apresentaram mortalidade hospitalar de 22\%, comparada com 1,4\% naqueles com função renal normal. Sadeghi et al., ${ }^{6}$ demonstraram que pacientes com disfunção renal em qualquer momento da internação hospitalar pós-ICP primária tiveram aumento significativo na mortalidade em 30 dias $(R R=13,8$; IC 95\% 7,3-26,2) e em 1 ano $(R R=7,4$; IC 95\% 4,7-11,7). Alguns autores descrevem que esse aumento de mortalidade persiste mesmo após longo tempo de observação. Amin et al. ${ }^{20}$ relataram que a piora da função renal foi associada a elevado risco de óbito em pacientes com IAM mesmo após 4 anos (HR = 1,64; IC 95\% 1,23-2,19). Um aumento de mortalidade também foi observado em pacientes com insuficiência renal crônica prévia ao procedimento. Gruberg et al. ${ }^{21}$ relataram que pacientes que apresentaram um incremento de $25 \%$ em sua creatinina basal tinham mortalidade hospitalar de 14,9\% comparados com 4,9\% sem disfunção renal adicional.

Brown et al. ${ }^{11}$ demonstraram que tanto alterações transitórias quanto persistentes da função renal estão associadas com pior prognóstico a curto e longo prazos. Alguns estudos demonstram que até 45 a 50\% dos pacientes que desenvolvem disfunção renal podem não retornar para sua função renal normal entre 2 a 4 semanas de evolução. ${ }^{11,22} \mathrm{Wi}$ et al. ${ }^{22}$ evidenciaram que pacientes com disfunção renal transitória após ICP no IAM apresentaram, em 2 anos, menor risco de óbito ou diálise comparados àqueles com disfunção renal persistente $(17,9 \%$ vs. 34,1\%; $\mathrm{P}=0,013)$; Contudo, mesmo aqueles com disfunção renal transitória tiveram taxa de eventos maior comparada àqueles sem disfunção renal $(17,9 \%$ vs. $6,3 \% ; P<0,001)$.

Alguns estudos têm sugerido que pacientes com doença renal têm menor probabilidade de receberem terapias medicamentosas ou de se submeterem a revascularização coronária, comparados àqueles com função renal preservada. ${ }^{23,24}$ Além disso, pacientes que desenvolvem piora da função renal, apresentaram maior número de fatores de risco, em relação ao grupo de pacientes que manteve a função renal normal, como idade avançada, diabetes e insuficiência cardíaca prévia. ${ }^{20}$ Lazaros et al. ${ }^{19}$ observaram ainda que os pacientes que desenvolveram piora da função renal durante a internação apresentavam doença arterial coronária mais importante e menos completamente tratada, com maior 
grau de necrose miocárdica e maior ativação neuro-hormonal - fatores que podem contribuir para uma pior evolução desses pacientes.

Mehran et al. ${ }^{25}$ desenvolveram um escore para avaliar o risco de IRA após ICP, incluindo variáveis clínicas e do procedimento: hipotensão, balão intra-aórtico, insuficiência cardíaca congestiva, doença renal crônica, diabetes, idade > 75 anos, anemia e volume de contraste. No IAM, Wi et al. ${ }^{26}$ demostraram que o escore de risco de Mehran foi um importante preditor independente de disfunção renal persistente e eventos cardio e cerebrovasculares adversos tardios em pacientes que se submeteram à ICP.

Se o paciente apresenta fatores de risco, alguns autores sugerem a adoção de medidas preventivas para o desenvolvimento de disfunção renal. ${ }^{27}$ Uma das mais importantes é uma adequada hidratação. O protocolo mais efetivo ainda não está bem definido, mas um estudo mostrou que a infusão de salina isotônica, na velocidade de $1 \mathrm{~mL} / \mathrm{kg} / \mathrm{h}$, por 48 horas, foi associada à redução significativa na incidência de insuficiência renal $(3,7 \%)$ quando comparada à hidratação oral sem restrição (34\%). ${ }^{27} \mathrm{~A} \mathrm{~N}$-acetilcisteína, apesar de representar uma intervenção farmacológica de baixo custo e praticamente não ter efeitos colaterais, teve sua indicação contestada após os resultados negativos do estudo ACT, ${ }^{28}$ não sendo mais recomendada de rotina para prevenção de nefropatia após procedimentos percutâneos. Drogas nefrotóxicas também aumentam o risco de nefropatia, sendo recomendado suspendê-las por, pelo menos, 24 horas antes do procedimento, quando possível. Além disso, o uso de uma menor quantidade de contraste de baixa osmolaridade ou isosmolar está associado com menor incidência de complicações renais. ${ }^{29}$

\section{Limitações do estudo}

O presente estudo foi realizado em um único centro de referência no atendimento de cardiologia, de forma não randomizada e observacional. Além disso, o estudo apresentou limitações na avaliação do tempo de isquemia, não tendo sido possível a aferição do tempo porta-balão - variável importante, por sua associação com a mortalidade. Não foi realizado ainda o cálculo do clearance de creatinina, assim como não foi possível obter informações em relação à quantidade de contraste utilizada e dos fármacos com potencial para ocasionar lesão renal, sabidamente fatores preditores de IRA após procedimento com contraste iodado.

\section{CONCLUSÕES}

A ocorrência de disfunção renal aguda após intervenção coronária percutânea de pacientes com infarto agudo do miocárdio com supradesnivelamento do segmento ST é uma complicação frequente, sendo associada com aumento de mortalidade nesse grupo de pacientes. A idade avançada, a presença de doença renal crônica prévia ao procedimento, a apresentação clínica em Killip III ou IV e a necessidade de cirurgia vascular ou de transfusões sanguíneas foram os principais preditores independentes de insuficiência renal aguda em nosso estudo. O acompanhamento da função renal na fase aguda do infarto agudo do miocárdio pode contribuir significativamente para a estratificação de risco e tratamento desses pacientes.

\section{CONFLITO DE INTERESSES}

Os autores declaram não haver conflito de interesses relacionados a este manuscrito.

\section{REFERÊNCIAS}

1. Wright RS, Reeder GS, Herzog CA, Albright RC, Williams BA, Dvorak DL, et al. Acute myocardial infarction and renal dysfunction: a high-risk combination. Ann Intern Med. 2002; 137(7):563-70.

2. Smith GL, Masoudi FA, Shlipak MG, Krumholz HM, Parikh CR Renal impairment predicts long-term mortality risk after acute myocardial infartion. J Am Soc Nephrol. 2008;19(1):141-50.

3. Sorensen CR, Brendorp B, Rask-Madsen C, Kober L, Kjoller E, Torp-Pedersen C. The prognostic importance of creatinine clearance after acute myocardial infarction. Eur Heart J. 2002; 23(12):948-52.

4. Walsh CR, O'Donnell CJ, Camargo CA Jr, Giugliano RP, Lloyd-Jones DM. Elevated serum creatinine is associated with 1 -year mortality after acute myocardial infarction. Am Heart J. 2002;144(6):1003-11.

5. Shlipak MG, Heidenreich PA, Noguchi H, Chertow GM, Browner WS, McClellan MB. Association of renal insufficiency with treatment and outcomes after myocardial infarction in elderly patients. Ann Intern Med. 2002;137(7):555-62.

6. Sadeghi HM, Stone GW, Grines $\mathrm{CL}$, Mehran $\mathrm{R}$, Dixon $\mathrm{SR}$, Lansky AJ, et al. Impact of renal insufficiency in patients undergoing primary angioplasty for acute myocardial infarction. Circulation. 2003;108(22):2769-75.

7. Rich MW, Crecelius CA. Incidence, risk factors and clinical course of acute renal insufficiency after cardiac catheterization in patients 70 years of age or older: a prospective study. Arch Intern Med. 1990;150(6):1237-42.

8. Best PJ, Lennon R, Ting HH, Bell MR, Rihal CS, Holmes DR, et al. The impact of renal insufficiency on clinical outcomes in patients undergoing percutaneous interventions. J Am Coll Cardiol. 2002;39(7):1113-9.

9. Rihal CS, Textor SC, Grill DE, Berger PB, Ting HH, Best PJ, et al. Incidence and prognostic importance of acute renal failure after percutaneous coronary intervention. Circulation. 2002;105(19):2259-64.

10. Lameire N, Van Biesen W, Vanholder R. Acute renal failure. Lancet. 2005;365(9457):417-30.

11. Brown JR, Malenka DJ, DeVries JT, Robb JF, Jayne JE, Friedman BJ, et al. Transient and persistent renal disfunction are predictors of survival after percutaneous coronary intervention: insights from the Dartmouth Dynamic Registry. Cathet Cardiovasc Interv. 2008;72(3):347-54.

12. Granger CB, Goldberg RJ, Dabbous O, Pieper KS, Eagle KA, Cannon $\mathrm{CP}$, et al. Predictors of hospital mortality in the global registry of acute coronary events. Arch Intern Med. 2003; 163(19):2345-53.

13. Lanza e Passos R, Siqueira DAA, Silva JFA, Sá FCF, Costa Junior JR, Feres F, et al. Insuficiência renal aguda após intervenção coronária percutânea primária no infarto agudo do 
miocárdio: preditores e evolução clínica a longo prazo. Rev Bras Cardiol Invasiva. 2008;16(4):422-8.

14. Marenzi G, Lauri G, Assanelli E, Campodonico J, Metrio M, Marana I, et al. Contrast-induced nephropathy in patients undergoing primary angioplasty for acute myocardial infarction. J Am Coll Cardiol. 2004;44(9):1780-5.

15. Piper WD, Malenka DJ, Ryan TJ Jr, Shubrooks SJ Jr, O'Connor GT, Robb JF, et al.; Northern New England Cardiovascular Disease Study Group. Predicting vascular complications in percutaneous coronary interventions. Am Heart J. 2003;145(6):1022-9.

16. Mehta RH, Parsons L, Rao SV, Peterson ED. Association of bleeding and in-hospital mortality in black and white patients with ST-segment-elevation myocardial infarction receiving reperfusion. Circulation. 2012;125(14):1727-34.

17. Dall'Orto CC, Willi LF, Nogueira MSF, Lapa GA, Oliveira Neto JB, Mauro MFZ, et al. Incidência, preditores e impacto clínico dos sangramentos maiores associados à intervenção coronária percutânea. Rev Bras Cardiol Invasiva. 2008;16(4):439-44.

18. Prada-Delgado O, Estevez-Loureiro R, Calvino-Santos R, Barge-Caballero E, Salgado-Fernandez J, Pinon-Esteban P, et al. Renal insufficiency and vascular complications after primary angioplasty via femoral route: impact of vascular closure devices use. Rev Esp Cardiol. 2012;65(3):258-64.

19. Lazaros G, Tsiachris D, Tousoulis D, Patialiakas A, Dimitriadis $\mathrm{K}$, Roussos D, et al. In-hospital worsening renal function is an independent predictor of one-year mortality in patients with acute myocardial infarction. Int J Cardiol. 2012;155(1):97-101.

20. Amin AP, Spertus JA, Reid KJ, Lan X, Buchanan DM, Decker C, et al. The prognostic importance of worsening renal function during an acute myocardial infarct on long-term mortality. Am Heart J. 2010;160(6):1065-71.

21. Gruberg L, Mintz GS, Mehran R, Gangas G, Lansky AJ, Kent $\mathrm{KM}$, et al. The prognostic implication of further renal function deterioration within $48 \mathrm{~h}$ of interventional coronary procedures in patients with pre-existent chronic renal insufficiency. J Am Coll Cardiol. 2000;36(5):1542-8.
22. Wi J, Ko Y, Kim J, Kim B, Choi D, Ha J, et al. Impact of contrast-induced acute kidney injury with transient or persistent renal dysfunction on long-term outcomes of patients with acute myocardial infarction undergoing percutaneous coronary intervention. Heart. 2011;97(21):1753-7.

23. Berger AK, Duval S, Krumholz HM. Aspirin, beta-blocker and angiotensin-converting enzyme inhibitor therapy in patients with end stage renal disease and an acute myocardial infarction. J Am Coll Cardiol. 2003;42(2):201-8.

24. Gibson CM, Pinto DS, Murphy SA, Morrow DA, Hobbach $\mathrm{HP}$, Wiviott SD, et al. Association of creatinine and creatinine clearance on presentation in acute myocardial infarction with subsequent mortality. J Am Coll Cardiol. 2003;42(2): 1535-43.

25. Mehran R, Aymong ED, Nikolsky E. A simple risk score for prediction of contrast-induced nephropathy after percutaneous coronary intervention: development and inicial validation. Am Coll Cardiol. 2004;44(7):1393-9.

26. Wi J, Ko Y, Shin D, Kim J, Kim B, Choi D. Prediction of contrast-induced nephropathy with persistent renal dysfunction and adverse long-term outcomes in patients with acute myocardial infarction using the Mehran Risk Score. Clin Cardiol. 2013;36(1):46-53.

27. Mathew R, Haque K, Woothipoom W. Acute renal failure induced by contrast medium: steps towards prevention. BMJ. 2006;333(7567):539-40.

28. Berwanger O, Cavalcanti AB, Sousa AGMR, Buehler AM, Kodama AA, Carballo MT, et al. Acetylcysteine for prevention of renal outcomes in patients undergoing coronary and peripheral vascular angiography: main results from the randomized Acetylcysteine for Contrast-Induced Nephropathy Trial (ACT). Circulation. 2011;124(11):1250-9.

29. Trivedi HS, Moore H, Nasr S, Aggarwal K, Agrawal A, Goel $\mathrm{P}$, et al. A randomized prospective trial to assess the role of saline hydratation on the development of contrast nephrotoxicity. Nephron Clin Pract. 2003;93(1):C29-34. 